OPEN ACCESS

Edited by:

Kelly Beekman,

Fontys University of Applied Sciences,

Netherlands

Reviewed by:

Susan M. Brookhart,

Duquesne University, United States

Hui Yong Tay,

Nanyang Technological University,

Singapore

${ }^{*}$ Correspondence:

Judith Gulikers

judith.gulikers@wur.n!

Specialty section:

This article was submitted to

Educational Psychology,

a section of the journal

Frontiers in Education

Received: 30 July 2021 Accepted: 27 September 2021

Published: 07 October 2021

Citation:

Gulikers J, Veugen M and Baartman L (2021) What are we Really Aiming for? Identifying Concrete Student Behavior in Co-Regulatory Formative

Assessment Processes in the Classroom

Front. Educ. 6:750281.

doi: 10.3389/feduc.2021.750281

\section{What are we Really Aiming for? Identifying Concrete Student Behavior in Co-Regulatory Formative Assessment Processes in the Classroom}

\author{
Judith Gulikers ${ }^{1 *}$, Marijke Veugen ${ }^{1}$ and Liesbeth Baartman ${ }^{2}$ \\ ${ }^{1}$ Education and Learning Sciences, Wageningen University \& Research, Wageningen, Netherlands, ${ }^{2}$ Research Group Vocational \\ Education, Research Centre for Learning and Innovation, Utrecht University of Applied Sciences, Utrecht, Netherlands
}

Formative assessment has the potential to incite co-regulatory activities that foster student's development of self-regulatory skills. Teacher often intend to use formative assessment to foster self-regulation. However, this requires purposeful interaction between students and teachers. To improve co-regulatory formative assessment implementation, research and professional development needs to pay attention to what co-regulatory formative assessment look like. This participatory study explores if and how the process of formative assessment-described in five phases of the formative assessment cycle -facilitates teachers to explicate concrete student and teacher behavior in their formative classroom that intends to stimulate self-regulated learning. Teachers of a longitudinal formative assessment professional development project participated in three activities in which a variety of data was collected. The results show that using the five phases of the formative assessment cycle helps to concretize co-regulatory formative assessment behavior that relates to various phases of regulation. They also show that starting from a student perspective, explicating expected student behavior and using this to explicate related teacher behavior, challenges teacher's formative assessment intentions and implementations. The activities make teachers realize the importance of purposefully designing co-regulatory formative assessments. Teachers explicated examples describe actual student-teacher co-regulatory interactions in the formative classroom and they are found to differ in the extent to which they are teacher-directed or student-directed. This challenges our thinking on the co-regulation processes incited by formative assessments and how this can stimulate students to develop self-regulatory skills.

Keywords: formative assessment, co-regulation, self-regulation, professional development, participatory research 


\section{INTRODUCTION}

Formative assessment and self-regulation of learning have been increasingly studied in interplay (Nicol and Macfarlance-Dick, 2006; Andrade, 2010; Panadero et al., 2018). For a long time, formative assessment has been conceptualized as a didactical approach that has the potential to stimulate students to develop self-regulatory skills (Black and Wiliam, 1998; Perrenoud, 1998). While schools can have several reasons implementing formative assessment practices, like improving student test performance, increasing conceptual understanding, achievement or motivation (Black and Wiliam, 1998; Yin et al., 2008; Meusen-Beekman et al., 2016), many teachers and schools implement formative assessment because they aim to stimulate students to take more ownership of their learning and develop self-regulatory skills. Panadero et al. (2019) argue that for formative assessment to stimulate student's development of self-regulatory skills, students need to be actively engaged in the formative assessment process. Or, formative assessments should foster a co-regulation of learning (Allal, 2020; Andrade and Brookhart, 2020). Brandmo et al. (2020) argue, in their introduction of a special issue on this topic in the journal Assessment in Education: Principles, Policy and Practice, for more integration of research on formative assessment (FA) and (self-)regulation to strengthen our knowledge on its relationship and what this means in the classroom. This study builds on one of the suggested aeras for further research (Panadero et al., 2019), exploring with teachers what co-regulatory formative assessment in the classroom can look like, in terms of concrete student and teacher behavior.

\section{Formative Assesssment, Self-Regulation and Co-Regulation}

The processes underlying formative assessment (FA) and selfregulated learning (SRL) are highly related, both describing various phases to systematically work towards certain goals (Andrade and Brookhart, 2020), while being informed by different theoretical strands (Panadero et al., 2018) and characterized by different types of research. SRL relates to students setting their own goals, planning and monitoring their own learning towards these goals, and evaluate and revise their learning if needed (Allal, 2020). SRL originates in psychological theories of (meta-)cognition, motivation and development of student learning and regulation and is mostly theoretical in nature (Brandmo et al., 2020), conceptualizing the underlying (meta-)cognitive, motivational and behavioral processes resulting in various detailed models describing the processes underlying SRL. All these models agree on SRL consisting of a preparatory/goal setting-performance/ monitoring-and evaluation/revision phase (Panadero et al., 2018). In SRL, these phases have mostly been conceptualized as internal (cognitive, motivational, metacognitive) processes (Zimmerman and Schunk, 2011).

Formative assessment is defined as classroom activities in which goal-directed evidence of student learning is elicited, interpreted and used by teachers, learners, and/or their peers, to make better decisions about the next step in learning and/or instruction (Black and Wiliam, 2009). Formative assessment research, contrary to SRL, originates in educational theories on testing and evaluation, studying how classroom assessment can influence student learning and development. This research is characterized as being more participatory and closely related to and contextualized in classroom practice and teacher professional development (Brandmo et al., 2020). Formative assessment focusses on three main phases questioning "where is the learning going?"; "where the learner is right now?" and "how to get there?" (Hattie and Timperley, 2007). In the formative assessment field these phases originally are mostly conceptualized as regulated by an external source. That is, these were mostly regulated by the teacher and curriculum standards (Andrade and Brookhart, 2020).

In both SRL and FA research, there is an increased focus on the dynamic interactions between students and teachers in the formative assessment process and in all phases of regulation (Wiliam et al., 2014; Van der Kleij et al., 2019; Andrade and Brookhart, 2020), stressing an active role for both teachers and students. In the field of formative assessment, Sadler (1989) seminal paper initiated the debate on the active student role in formative assessment. Stiggins et al. (2004) proposed that formative assessment covers seven teacher strategies, of which three directly refer to actively engaging students in self-regulatory activities: Teach students to self-asses and set goals; teach students focused revision, and engage students in selfreflection and let them keep track of and share their learning. Boud and Molloy (2013) were the first to explicitly conceptualize formative assessment as an educational design process in which teachers should purposefully design the learning environment in such a way that this actively engages students in the formative assessment process. If teachers intend to use formative assessment to stimulate self-regulation, this implies that teachers should incorporate SRL activities, relating to all SRL processes, within their formative assessment design (Andrade and Brookhart, 2020).

To bridge the gap between the internal processes of selfregulated learning and external processes of formative assessment, Allal (2020) proposes a new framework of coregulated learning to illustrate that student's self-regulation is always influenced by the regulatory activities incited by the learning environment or curriculum, teacher activities, and tools. Allal (2020) defines co-regulation as "the joint influence on student learning of the learner's processes of self-regulation and of the sources of regulation in the learning environment" ( $p$. 7). Formative assessment has the potential to foster powerful coregulatory activities in the classroom (Andrade and Brookhart, 2020) as long as teacher's formative assessment activities enhance rather than inhibit students to participate in this regulation process (Allal, 2020). However, empirical research shows that formative assessment strategies that foster student regulatory activities -such as self-assessment or peer-assessment (Wiliam. 2011)-are used the least in educational practice (Kippers et al., 2018; Brandmo et al., 2020). Moreover, several recent studies (Van der Kleij et al., 2019; Brooks et al., 2021) showed that it -still-is not a given that formative assessment practices enhance student's active role in this process. Thus, while formative 


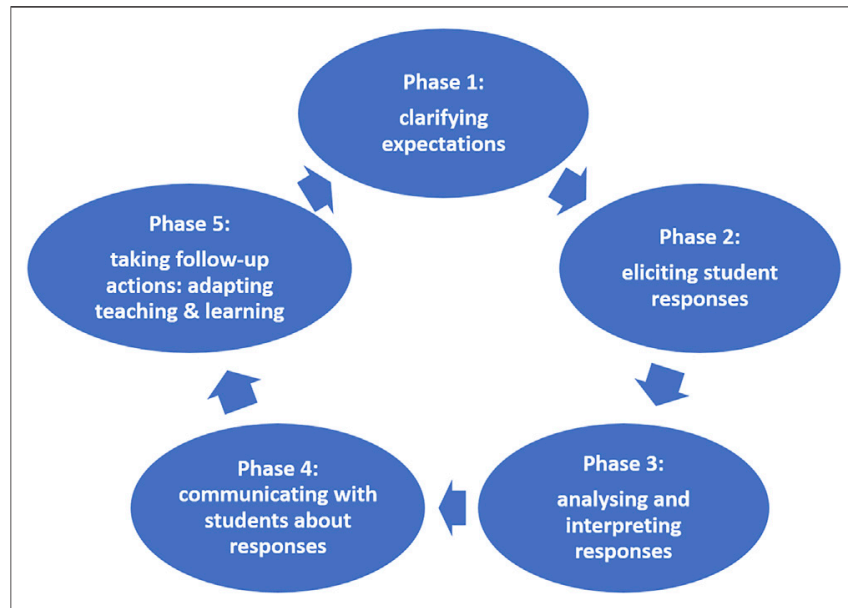

FIGURE 1 | The formative assessment cycle (Gulikers and Baartman, 2017; Veugen et al., 2021).

assessment often intends to stimulate SRL, and has the potential to incite co-regulatory activities that foster students' development of self-regulatory skills, not all formative assessment practices are automatically co-regulatory. The teacher should purposefully design co-regulatory formative assessment activities to enhance-and not inhibit-student's development of selfregulatory skills (Allal, 2020). The next section describes the theoretical framework-the formative assessment cycle-that is used in this participatory study to elicit teacher's thinking on co-regulatory formative assessment in terms of student and teacher behaviour in the classroom.

\section{The Formative Assessment Cycle}

This study uses the Formative Assessment Cycle (FA cycle, Figure 1; Gulikers and Baartman, 2017; Veugen et al., 2021) as an underlying theoretical framework to study co-regulatory formative assessment practices in classrooms. In line with arguments of Andersson and Palm (2018), this FA cycle describes the formative assessment process as an aligned set of five phases: 1) clarifying expectations (i.e., learning goals and success criteria); 2) eliciting responses of student learning; 3) analyzing student responses; 4) communicating about results (including feedback); and 5) taking concrete actions to adjust teaching and/or learning. Ideally, students and teachers are both active and in interaction in all phases, thus co-regulating student learning. Previous studies using this FA cycle focused on teacher behavior (Gulikers and Baartman, 2017; Veugen etal., 2021) from the idea that the teacher needs to design a learning environment in such a way that it stimulates and facilitates students to engage in the formative assessment process (Carless and Winstone, 2020) and enhance student's self-regulatory skills (Allal, 2020). These studies typified concrete teacher behavior in the classroom within these five phases based on a review of 106 scientific studies (Gulikers and Baartman, 2017) and an empirical study examining teacher practices in the classroom (Veugen et al., 2021). These studies showed the FA cycle to be a valid and practical framework for studying teacher's FA behavior in the classroom, also pinpointing to strengths and weaknesses. Teachers were found to score lowest on the activities that were geared towards actively involving students within these five phases (i.e., items like "I let student analyze their own work" or "I let peers discuss possible follow up activities" (Veugen et al., 2021). To improve co-regulatory formative assessment implementation, research and professional development needs to pay attention to what co-regulatory classroom practices look like (Panadero et al., 2019; Brooks et al., 2021).

\section{The Context of this Study: Participatory Research in a Learning Network}

This study is contextualized in a longitudinal-4 year-formative assessment professional development project (i.e., the FA learning network) in which teams of teachers and school leaders from secondary schools discuss, develop, experiment with, reflect on and learn to implement the formative assessment process in their classroom in collaboration with researchers and assessment experts (McDowell et al., 2008; Veugen et al., 2021). Within this learning network we use the FA cycle as theoretical framework to facilitate these processes and create a shared understanding. In this learning network, teacher professional development and research on formative assessment are intertwined. It uses a participatory action research approach (Jacobs, 2016) in which teachers questions-resulting from experimenting with the theory of the formative assessment cycle-are guiding the activities and research questions in the learning network.

To be able to participate in the learning network, schools had to have formative assessment as a focal point of their school vision. In their vision all schools intended to make use of more formative assessments in their classrooms for the purpose of stimulating students as owners of their own learning and regulating their own learning. The learning network started in April 2018 and up till this study (January 2020), the focus has been on teacher behavior. After one-and-a-half year of experimenting with the FA cycle in their classrooms, teachers increasingly started to ask questions about their student's behavior: What is it actually that we want our students to do in our formative classrooms? One school leader phrased it explicitly as "it is our shared vision to use formative assessment to stimulate students to regulate their own learning, however, we as team do actually not agree on or have a concrete idea of what this actually means in terms of student behavior in the formative classroom". These questions led the researchers to develop an intervention for teams of teachers to elaborate on this research question using the FA cycle to reflect on their expected student behavior (see Methods section) and using this student perspective to reflect on the student-teacher-coregulatory-formative assessment activities in their classroom.

Our research tries to provide further knowledge of what coregulatory formative assessment in the classroom can look like by starting from the perspective of (expected) student behavior (Andrade and Brookhart, 2020). It asks the question of what concrete student behavior teachers expect to see in their formative classroom that intends to incite student's self- 
regulatory activities. Second, it aims to explore if explicating this expected student behavior in turn helps teachers to also identify teacher behavior that is needed to elicit this expected student behavior. By exploring and explicating this expected student behavior together with teachers and linking this to their teacher behavior, this study intends to contribute to teacher's practical knowledge on how to design for co-regulatory formative assessment processes that stimulate student's self-regulatory skills in their classrooms Research questions are:

1) How do secondary school teachers describe the formative assessment behavior they expect from their students within their formative classroom intended to stimulate SRL, using the five phases of the FA cycle? and

2) What examples of concrete co-regulated FA practices do the teachers describe when linking their expected student behavior to their own teacher behavior using the FA cycle?

\section{METHODS}

This study was part of the 4 year professional development project (i.e., learning network) in which teacher teams of 17 different secondary schools in the Netherlands participated, with in total 132 teachers and 25 school leaders. Teachers taught in different grade level (for students from 12 to 18 years old) and various school subjects being language (Dutch, English, German, French), biology, physics/chemistry, geography, history and arts. Researchers and assessment/curriculum experts organized and facilitated the learning network activities. These consisted of three plenary meetings with all teachers and two school-based meetings per year. This study is an explorative, participatory study in which researchers and teachers from the FA learning network collaborated.

In participatory action research (Jacobs, 2016) the research is conducted together with the participants by seeking for solutions to their problems, experimenting in their own context, constructing knowledge from their experiences and collaboratively with researchers reflecting on research findings, which results in rich explanations and interpretations of the data (Jacobs, 2016). First, knowledge of what teachers seek in FA student behavior was created, with learning network activities aimed at explicating concrete, observable student behavior in the FA process that intends to stimulate SRL (RQ 1). Second, discussing these empirical findings and reflecting on them using theoretical insights on formative assessment as a coregulatory process, an additional learning network activity was undertaken. This focused on linking expected student behavior to required teacher behavior, showing concrete examples of what co-regulatory formative assessment practices in the classroom can look like (RQ 2.) The participatory study had three steps, of which the first two steps aimed to answer research question 1 and the third step to answer research question 2.

Step 1: student FA cycle posters. In total 24 teams of teachers, from 15 secondary schools participating in the FE learning network, took part in this first step that was conducted during one of the plenary meetings of the learning network (January
2020). A poster assignment and poster market activity were undertaken. Teacher teams were asked to draw their own Formative Assessment Cycle with five phases on a flip-over page, and write down as much as possible concrete and observable student behaviors per phase. They were asked to describe the "self-regulating student" or "the student who takes ownership of his/her learning" in as concrete as possible observable actions. In an informal poster market the teams compared, reviewed and discussed each other's posters focusing on the explicated student behavior. They gave each other feedback and noted down some points for inspiration for their own poster. After having done so, a plenary discussion was held to share some lessons learned in terms of what insights this activity gave teachers for making student behavior in formative assessment aimed to stimulate SRL more tangible. Two researchers made field notes during this plenary evaluation, which were also used as data input for this study.

Step 2: Interactive workshop explicating student behavior. During an online conference of the learning network (November 2020) 27 secondary school teachers from the learning network participated voluntarily in an online workshop (note: This step was conducted during the COVID19 lock-down period). The five phases of the FA cycle were again used to stimulate teacher's thinking about this expected student behavior. Within this workshop, teachers added as concrete as possible ideas on expected student behavior in the five phases of the FA cycle on a shared padlet (i.e., digital bulletin board; www. padlet.com). For every phase of the FA cycle a separate column was created on the padlet. At the end of this workshop, a plenary evaluation was held and 15 teachers shared their lessons learned, main new insights and remaining questions regarding the formative assessment process in their classroom via an exit ticket (www.exitticket.nl). Field notes, padlet posts and exit ticket answers were used as data input for this study.

Step 3. Developing student-teacher FA cycles. After analysis of the results of steps 1 and 2, an additional in-depth workshop was held in the spring of 2021 with 11 voluntary teachers of 4 schools of the learning network. This workshop focused mainly on research question 2. This workshop again stimulated teachers to explicate expected student regulatory FA behavior by using the FA cycle phases (research question 1). Contrary to step 1 and 2, this workshop focused on linking this expected student behavior to teacher behavior that can elicit this regulatory student behavior in the classroom, and thus, exemplify co-regulatory formative assessment practices (research question 2). In this workshop we elaborated the empirical findings of step 1 and 2 with the theoretical perspectives of Boud and Molloy (2013), Carless and Winstone (2020) and Allal (2020). Showing teachers that they should make purposeful decisions in designing co-regulatory formative assessment in order to actively engage students and stimulate SRL. The key messages discussed here with teachers were 1) formative assessment is a dialogical process, that requires purposeful interaction between students and teachers; 2) formative assessment is an educational design challenge. This means that teachers have to explicitly think about how to design their education in such a way that this prompts these purposeful interactions and the (regulatory) behavior they expect from 


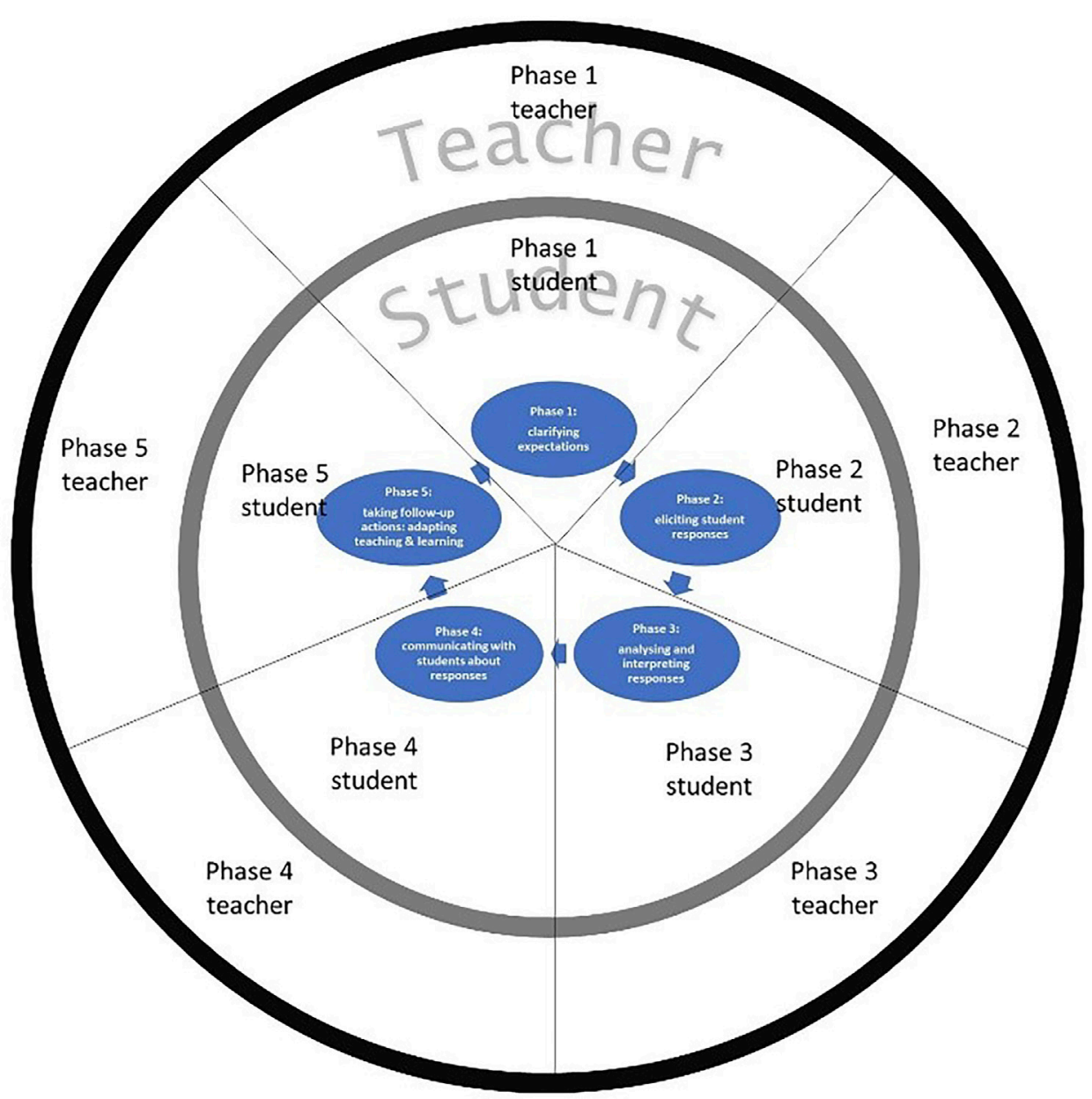

FIGURE 2 | The student-teacher co-regulatory formative assessment cycle format.

students; 3) the concept op co-regulation of learning can help to think about how teachers formative assessment activities can foster, prompt or scaffold the self-regulatory activities of students. The first author facilitated the session and discussions, while the second author moderated and took notes. In this workshop the same padlet assignment was used as in step 2 to elicit teacher's ideas on concrete observable student behavior per FA phase, aimed at stimulating SRL, and link this to related teacher behavior. Building on their posts on the padlet, the facilitator stimulated teachers to give examples of student-teacher interactive (i.e., co-regulatory) formative assessment activities in their classrooms. Then, the results of step 1 and 2 were explained by the facilitator and discussed with participants, prompting their ideas on formative assessment as being a coregulatory process between students and teachers. After this, teachers were asked to exemplify concrete co-regulatory formative classroom interactions using the FA cycle. For this they were provided with a format of the FA cycle to explicate specific students and related teacher behavior per FA phase (See Figure 2). After the session they were asked to fill it in with a team of teachers in their own schools during physical team meetings with their colleagues at school (i.e., secondary schools were at this time not in full COVID-lockdown anymore). This resulted in seven student-teacher FA cycles from 7 different teams of teachers (3-4 teachers per team, in total 22 teachers). The online discussion was video recorded with informed consent from all participants and, together with the teachers' created FA cycles and field notes, used as data for this study.

\section{Data Analysis}

All data on student behavior were analysed together. All statements of the posters (step 1) and the padlet (step 2 and 3) were collected per phase of the FA cycle. Every individual statement written down by a teacher team or individual teacher was treated as one fragment. Bottom-up coding was used in several rounds by two independent researchers (see Table 1). During first cycle coding (Miles et al., 2020) both researchers independently reviewed all fragments, to create codes that helped to grasp how teachers talked about their expected student behavior. Discussion amongst the researchers resulted in a set of codes that allowed for counting fragments and/or categorizing the fragments into more meaningful themes. These codes were: 
TABLE 1 | Overview of analysis steps, codes and themes.

Themes overarching the

five FA phases

Concrete

Fitting the right FA phase $\quad Y e s / n o$

(not) Fitting FA

Student or teacher behavior

Yes/no

\section{Quantitative} analysis round 1

Student/teacher

Qualitative

analysis round 2
Additional

subthemes round 2

- Type of interaction

- Student or teacher directed

- Key intention

- Lack of alignment

- Active student

- Culture of mistakes

TABLE 2 | More and less concrete examples of student FE behavior.

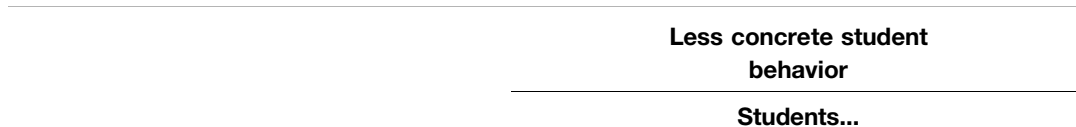

Phase 1 Clarifying expectations
Understands the learning goals and success criteria

\begin{tabular}{c}
$\begin{array}{c}\text { More concrete student } \\
\text { behavior }\end{array}$ \\
\hline Students...
\end{tabular}

Ask questions about the learning goals

Compare different examples to deduce success criteria Collaboratively (with teacher) formulate success criteria

Formulate own learning goals

Help peers in understanding the learning goals and success criteria

Formulate success criteria in own words
Phase 2 Eliciting student responses take responsibility are active in class take the assignments seriously

Perform (self-chosen or teacher provided) task to show to what extent they have reached the learning goals

Give answers and explanations

Selects from a number of provided assignments to show his/her current performance

Design their own assignments by which they want to show the learning goals

Explain "wrong" examples

Explicate their current prior knowledge

Phase 3 Analysing and interpreting responses Look at their own or each other's work Are critical towards their own work Accept mistakes
Identifies own strength and weaknesses

Can explain and justify their provided answers and reasoning Compare own work using the success criteria Compare their work with that of peers

Give their own performance an indicative mark Checks the degree to which they have met the learning goal by using the provided answer models

Ask themselves the question "To what extent do I fulfil the criteria?"

Identify mistakes in their own work

Give each other feedback using the success criteria Ask for feedback

Answer for themselves "how far along am I?"

Discuss with the teacher what they have done well and what needs to improve

Discuss received feedback with the teacher or peers
Phase 5 Taking an concrete follow up action in Know what they should do differently Make feedback instruction and/or learning
Choose an appropriate follow up task, if needed in collaboration with the teacher Asks the teacher for additional instruction Articulates "what do I need to take a next step?" Adjusts product using the received feedback Writes down what next steps to take Actively search for people who can help them reach their goal Asks for a new assessment opportunity Move on to making a more difficult task Ask help from peers 
concrete (yes/no and more/less concrete in terms of student observable behavior), fitting the right FA phase (yes/no), fitting FA at all (yes/no), and teacher or student behavior (several fragments described teacher behavior instead of student behavior). With these codes the data were reviewed again, to count the number of not concrete statements, not fitting FA/FA phase, and teacher instead of student behavior. This first quantitative round was done across the five FA phases and used to grasp and clean the data. The not fitting FA statements and the statements describing teacher behavior were deleted. Then, all remaining fragments on FA student behavior were categorized by both coders into the right FA phase after which more meaningful qualitative analysis was possible. Second cycle coding by both researchers resulted in identification of themes that typified the variation of expected student behavior for all FA phases. Per FA phase, the statements were coded as 1) more or less concrete examples of student coregulatory behavior; 2) teacher-student directedness activities (who is steering the regulating processes?), and 3) type of interactions (student, peers, teacher-student). These results typify the variety of student behavior per FA phase that aimed for stimulating SRL, in the eyes of teachers (see Table 2, results section). Four additional themes were deduced overarching the specific FA phases: Key intention; lack of alignment; active student, and culture of mistakes. These themes might point to underlying categories facilitating or hampering a co-regulatory formative assessment process.

For research question 2, data analysis focused on the in-depth workshop data. First, the padlet, the video recording, the field notes and the seven student-teacher FA cycles teacher teams made as a follow up of the in-depth workshop (step 3) were analyzed by both researchers independently. This analysis aimed to identify all student-teacher co-regulatory formative interactions per FA phase that were mentioned during the meeting. Second, the seven student-teacher FA cycles were reviewed by both researchers to identify themes that demarcate differences in these co-regulatory formative assessment examples across the whole FA process. The agreed upon themes were: level of detail, alignment, use of peers, task and/or learning strategy oriented, and student versus teacher directed (i.e., who is steering the activities?). Finally, from these examples, exemplary co-regulatory student-teachers formative assessment interactions were selected to make more tangible what co-regulation processes in the classroom can look like and what this means for both teachers and students, both per FA phase and across the whole FA process.

\section{RESULTS}

\section{Reserach Question 1: Student Behavior in Co-Regulatory FA-Activities}

In total, 420 statements were identified. The first round of coding was done across these 420 statements representing the five FA phases. Of these statements, $22 \%$ were placed in the wrong FA phase, like "I use feedback of the teacher" in phase 2 (eliciting responses) where this should be phase 5 (taking a follow-up action). Some statements were coded by both researchers as not FA behavior when there was no direct link to any of the five FA phases. These were statements like "student collects his things" or "student has the preconditions in order". These results showed that not all teachers fully understood the FA cycle and it phases.

About $33 \%$ of the statements did not describe concrete and observable student behavior, indicating that teachers might find this difficult. Examples were: "the student knows/understands/ thinks about ... (learning goals)" or "is aware" or "needs to get an overview of his/her ownership of the learning process", or more attitude-oriented statements like "curiosity" or "shows a learning attitude". Moreover, about $10 \%$ of all statements were coded by both researchers as teacher instead of student behavior, such as "I let students think about their learning goals" or "I check their prior knowledge". While these statements were important in terms of co-regulation, they did not align with the assignment of describing as concrete as possible student behavior. The fragments that were coded as not FA behavior or teacher instead of student behavior were deleted before going to the next round of qualitative analysis.

The qualitative coding resulted in a categorization of all student statements per FA phase, identifying more or less concrete and observable student co-regulatory FA-behavior per FA phase. Table 2 shows a variety in teacher's statements about their expected student behavior per FA phase, categorized as more or less concrete. Within the more concrete category, two additional themes were identified. First, different types of interaction showing statements referring to individual student behavior (formulate success criteria in their own words), studentteacher interactive behavior (formulate success criteria collaboratively with the teacher) and peer interaction (help peers in understanding the learning goals). Second, student or teacher-directedness: They showed examples of more teacherdirected activities (students perform a teacher provided task to show to what extend they have met the learning goals) or more student-directed activities (students design their own assignments to show the learning goals).

Four themes were identified overarching the five FA phases (see Table 1). First, it was noted that 7 out of the 24 posters had a key intention or vision of FA in the middle of their student FA cycle. These intentions differed slightly. For example, one school wrote "the active student", another school "I have an open and honest learning attitude", "under construction" or "growth mindset". These teams started the development of their student FA cycle by discussing their overarching intention with formative assessment in terms of the self-regulated student: "For us, this self-regulated student wants to learn and develop" (open and honest learning attitude) or "students believe that they can always improve" (growth mindset). Other teams did not place something in the middle and did not started the development of their student FA cycle with this overarching discussion. Second, almost all posters referred to learning goals and success criteria and to students collaboratively designing or doing something with these learning goals/success criteria in FA phase 1 (clarifying expectations). However, the described student activities in phase 2, 3, 4 or 5 often did not refer to these learning goals, suggesting a lack of alignment within the regulatory FA 
process. For example, many statements of student behavior in the analysis phase (FA phase 3) or feedback phase (FA phase 4) did not link to the learning goals/success criteria, while both in the FA as well as SRL process, revisiting the learning goals is prerequisite. The third and fourth theme were identified as characterizing student behavior statements across the five FA phases. The two themes were student showing an active attitude or a classroom culture of learning from mistakes. Student's active attitude was expressed in statements such as: Students search for eye contact, are actively working on their task, ask questions, ask for help, ask for feedback, explain their reasoning. The classroom culture of making mistakes was shown in statements such as: Students dare to express their mistakes, students take advantage of their mistakes, students give honest answers also when not sure. These two themes might show two classroom conditions that need to be put in place for students to take up their role in the coregulatory formative assessment process.

\section{Research Question 2: Student-Teacher Co-Regulatory Formative Assessment Behavior}

This section addresses research question 2 on student-teachers co-regulatory formative assessment examples. For this, the padlet and video recording data from the in-depth workshop (step 3) were used as well as the student-teacher FA cycles the seven teacher teams developed as a follow up activity of this in-depth workshop. First, the FA phases were used to describe a variety of concrete examples of student-teacher co-regulation formative assessment in the classroom. Then, analysis of the seven student-teacher FA cycles resulted in the identification of seven themes that differentiated the ways the teams of teachers described student-teacher co-regulation in the formative classroom. Finally, the most elaborated and worked out student-teacher formative assessment cycle, developed by one teacher team, is presented to exemplify what student-teacher coregulation in the whole formative assessment process can look like.

A first example referred to FA phase 1 (clarifying expectations). This discussion focused on whether or not students need to define their own learning goals (phase 1 activity). The discussion of the teachers of the in-depth workshop explicated a realization in this respect:

If we want our students to be able to regulate their own learning, they need to be able to set some goals for themselves. However, this might be more of a phase 5 activity (i.e., taking a follow-up activity) as students will not be able to define their own learning goals if they have no prior knowledge on the topic. We first have to go through the FA cycle once, where we as teachers set the learning goals (phase 1 activity). After having gone through a FA cycle, then students have grasped the ideas of the learning goals and success criteria (phase 1 activity), their own performance and strengths and weaknesses in this respect (phase 2-3-4 activities) that allow them to identify personal follow-up goals to work on in a next task (phase 5 activity).

The second example referred to FA phase 3 (analyzing responses), and showed teacher's realization of the mutual responsibility in co-regulatory formative assessment process. Many teachers noted down, on the padlet, student behavior in FA phase 3 (analyzing student responses) as "students evaluate each other's work using the success criteria". Reviewing this expected student behavior in the follow-up discussion during the in-depth workshop led teachers to say:

If I want my students to do this, this means that I 1) have to undertake an activity to let students know and understand the success criteria (phase 1 activity); 2) give students an assignment in which they can show their performance of these success criteria (phase 2 activity), and 3) give then a format, tool and time to actually compare each other's work using these success criteria (phase 3 activity).

One teacher made this example even more clear:

In phase 3 ("analyzing student responses"), we want our students to evaluate their own work, using the success criteria of phase 1 . I noticed in my classroom that students cannot automatically do this. So I learned to model this process. I reviewed one piece of student work in the (online) classroom. After having done so, I asked all student to identify three criteria they noticed me using for analyzing this piece of work and enter this in a mentimeter (online tool) creating a work cloud of their criteria. This word cloud then showed them the collaboratively identified criteria for reviewing their own and each other's work. This allowed them to analyze their own work using these criteria afterwards. (Mike, Science teacher. this example is contextualized in the online teaching period of the Covid-lockdown)

This discussion suggested that thinking of concrete student behavior gave teachers new ideas about concrete teacher behavior and the importance of taking these student expected student behaviors into account in the design of their formative assessment activities in the classroom.

The final example referred to FA phase 5 (taking a follow up action). Teachers discussed how to make "taking a follow up action in learning" more concrete. Teachers discussed the challenge of letting students think about alternative learning strategies. One teacher said (John, French teacher):

I like to have the discussion with students on how they have learned for a certain task or goal, however, I mostly get an answer like "I don't know, I just do as I always do". So, students seem to be not aware of their learning strategy, making it also difficult to change. 
Another teacher responded with her approach (Maria, Nursing Teacher)

I like to flesh out student's approach to learning when a student has been very successful. I ask this student-in the plenary classroom-"How did you approach this task?” Obviously I often need to ask several follow up questions to help to student to more concretely explicating his/her learning approach, but this often results in concrete insights in the approach to learning that was effective for this student and this task. I like to stress these positive examples and also notice that this motivates students. (She follows up by saying ...) I have been doing this for a some time now, and now I get to the point where I can ask students to share in peers how they have approached a task and what was successful or not. I notice that this has more impact on them, then when I tell them what approach to take.

After the workshop, the teachers of the in-depth workshop took the student-teacher FA cycle format (see Figure 2) to their teacher teams to discuss and describe as concrete as possible student-teacher co-regulatory behavior within the whole FA process. Analysis of the resulting seven student-teacher FA cycles illuminated six themes that characterized differences in the co-regulatory formative assessment processes in the classroom as described by the teacher teams. They differed in level of detail with more detailed versions describing more concrete student and teacher activities. Comparable to the finding on more or less concrete student behavior in Table 2, these results also showed more or less concrete teacher behavior within all phases of the formative assessment process. Examples of less concrete teacher behavior were: "Collects responses", "guides the process" or "stimulates learning behavior". Examples of more concrete teacher behavior were: "Let's students think about what quality looks like", "provides students structure for giving each other feedback", "discusses with students how they can improve their performance". Second, the student-teacher FA cycles differed in the extent to which the student-teacher activities seemed to describe an aligned process, in terms of alignment between the five FA phases and between student and teacher activities. In some cases the activities indeed logically followed up on one another, where in other instances they seemed to describe disconnected-or seemingly unrelatedactivities across FA phases, thereby not showing an aligned coregulation process. Contrary to the findings in RQ1, where teachers only looked at describing expected student behavior, the results of these student-teacher FA cycles showed that the learning goals mentioned in phase 1 (e.g., the teacher explains the learning goals), were more often revisited in the other FA phases. For example, "The student reflects on the extent to which the learning goal is met" (phase 4, feedback) or "the student selects additional assignments related to learning goals that are not met yet" (phase 5, taking follow up action). Third, the most important difference in the seven described student-teacher FA cycles was coded as student vs. teacher directed (i.e., who is steering the activities?). While in all seven examples, the teachers provided the learning goals (FA phase 1) and the student showed some kind of active behavior, the activities differed from being largely teacher directed to being more student directed. In the teacher-directed examples, the teacher decided what activities the student needed to undertake. For example, the teacher provides the tasks to make, gives feedback, tells students what to do with the feedback. On the other hand, in the student-directed examples, the student seemed to be the initiator of activities and more focus was on studentteacher dialogue. For example, success criteria are developed in dialogue, the student decides what tasks to undertake, students analyze own work, the student asks for feedback. Finally, the student-teacher FA cycles differed in extent which they made use of peers in the various FA phases (analyzing each other's work, giving peer feedback, asking peers for help in identifying a next step), addressed task performance (the student improves his/her work) and/or addressed learning strategy (the student evaluates his/her chosen learning strategy; in dialogue with the teacher the student identifies what learning approach worked for him/her). The more student-directed examples seemed to make more use of peers in different FA phases, and more explicitly referred to the learning strategy next to improving task performance, where the more teacher-directed examples focused on improving task performance (student takes the diagnostic test; student improves the assignment).

To give a final example of co-regulation between students and teachers in the formative classroom, Figure 3 shows the most detailed student-teacher FA cycle developed by one of the teacher teams. This is exemplary, and not extensive or exclusive, but showed how using the FA cycle allowed teachers to explicate concrete student-teacher interactive behavior to exemplify what the various phases of co-regulation in the classroom can look like.

\section{DISCUSSION}

Formative assessment can and should play a central role in regulation of learning processes (Perrenoud, 1998; Nicol and McFarlance-Dick, 2006; Clark, 2012). It has the potential to incite co-regulatory activities that foster student's development of selfregulatory skills (Allal, 2020; Andrade and Brookhart, 2020). However, this requires purposeful interaction between students and teachers (Carless and Winstone, 2020). To improve coregulatory formative assessment implementation, research and professional development needs to pay attention to what coregulatory classroom practices look like (Panadero et al., 2019; Brooks et al., 2021). This participatory study explored if and how the process of formative assessment-described in five phases of the formative assessment cycle (Gulikers and Baartman, 2017; Veugen et al., 2021)-facilitates teachers to explicate concrete student and teacher behavior in their formative classroom that intends to stimulate self-regulated learning. The results show that taking a student perspective, that is, starting to think about the coregulatory formative assessment process from the perspective of concrete student behavior that teachers expect to see in the classroom, sheds new lights on making co-regulation in the formative classroom more tangible. Using the five phases of the formative assessment cycle helps to concretize co- 


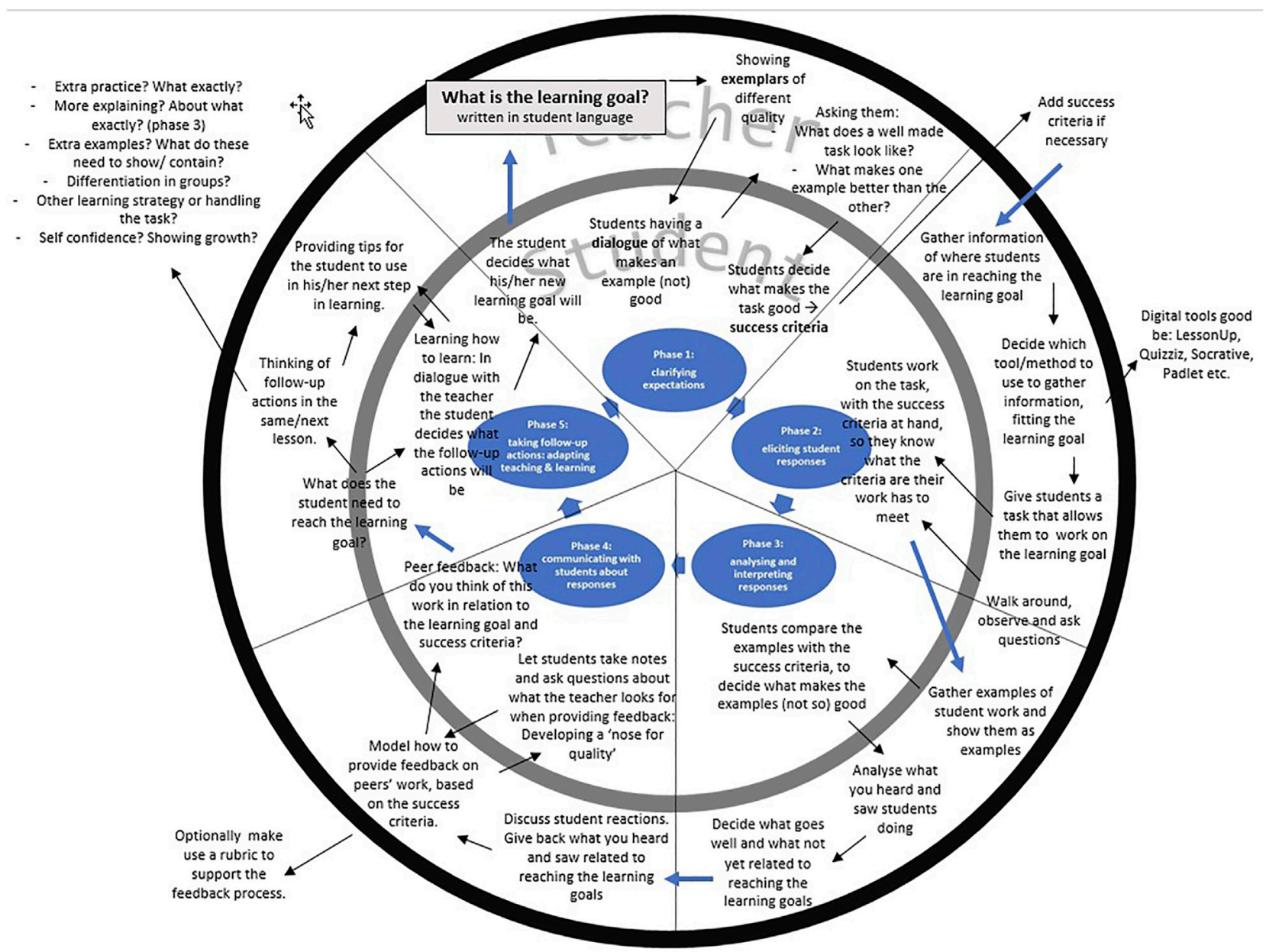

FIGURE 3 | An exemplary student-teacher co-regulatory formative assessment process.

regulatory formative assessment behavior that relates to various phases of regulation. Second, this student perspective, in turn, facilitates teachers to also concretize their own co-regulatory behavior in their formative assessments. These activities make teachers realize the importance of purposefully designing coregulatory assessment activities that incite these expected regulatory behaviors of students (Boud and Molloy, 2013; Winstone and Carless, 2019). Finally, the added value of the examples of concrete co-regulatory formative assessment practices, described by teachers in this study, is twofold. First, they describe actual student-teacher co-regulatory interactions in the classroom and second, they differ in the extent to which they are teacher-directed or student-directed. This challenges our thinking on the co-regulation processes incited by formative assessments and how this can stimulate students to develop self-regulatory skills. This discussion will first elaborate on these three insights. Followed by a reflection on the found indicators that might facilitate implementing co-regulatory formative assessment. Lastly, some limitations of this study are discussed. Incorporated in these discussions we will also reflect on the value of the participatory activities of this study for elevating teachers practical knowledge on co-regulatory formative assessment for stimulating student's SRL. These insights can be taken into account in future, participatory, research and professional development.

\section{The Active Student in the Co-Regulatory Formative Assessment Process}

Building on a future research suggestion of Andrade and Brookhart (2020), who conceptualized formative assessment as the co-regulation of learning, we argue that to help actual implementation of co-regulatory formative assessment in the classroom more concrete handles as to what kind of student behavior teachers intend to stimulate in the formative classroom would be helpful. Therefore, this participatory study starts from a student perspective to identify what co-regulatory formative assessment in the classroom should look like using the formative assessment cycle to help teachers to explicate expected student behavior in the various phases of the formative assessment process. The results suggests that taking this student angle, combined with the five phases of the formative 
assessment cycle, was indeed insightful. It allows teachers to make their FA intentions of "stimulating students to take ownership of their learning" or "to self-regulate their learning" more clear and practical. While teachers found it challenging to describe concrete student behavior, a joined effort of 132 teachers in the FA learning network let to the identification of a variety of concrete student activities in all five phases of the formative assessment process (See Table 2). These student activities clearly link to SRL behavior in the phases of goal-setting, progress monitoring, and revision (Andrade and Brookhart, 2016). For example, clarifying expectations activities (FA-phase 1) relate to the goal setting phase: students ask questions about the learning goals or student collaborate with teachers to deduce the success criteria; activities of eliciting evidence (FA-phase 2), analyzing responses (FA-phase 3), and communicating about results (FAphase 4) relate to the process monitoring phase: student explain their answers, student compare each other's work to the success criteria, student discuss received feedback with the teachers; and activities related to taking a follow up action (FA-phase 5) relate to the revision phase: students articulate what they needs to more forward, asks a peer for help.

While Table 2 shows a range of concrete examples of student behavior in all five phases of the co-regulatory FA process, we want to draw specific attention to phase 5 of taking a follow-up action. Various studies showed that teachers find this follow-up phase the most difficult phase, as they lack time or a pedagogical repertoire to act upon gathered student responses (Heritage et al., 2009; Herman et al., 2015; Veugen et al., 2021). However, this follow up phase of FA-or the reflect and revise phase in SRL-is key to formative assessment. If the formative process does not result in a follow up, its formative or regulatory intention-to further learning-will not be met (Winstone and Carless, 2019). Contrary to teacher practices, Table 2 shows a variety of ideas for concrete student behavior in this follow up phase. Veugen et al. (2021) argue that this follow-up phase requires explicit and specific attention in teacher professional development. The intervention of this participatory study in which teachers are challenged to explicate concrete expected student behavior might give teachers more ideas on the variety of concrete behavior they could be thinking about in co-regulating this follow up phase of regulation within their formative assessment classroom.

\section{The Active Teacher in the Co-Regulatory Formative Assessment Process}

This study also shows that explicating student behavior helps teachers to make their own behavior in the co-regulatory formative assessment process more clear. Teachers in the indepth workshop explicitly discussed the mutual responsibility between student and teachers in the formative assessment process that intends to stimulate SRL. For example, regarding who should be the one to set the initial learning goals. Where SRL assumes students to set their own learning goals (Zimmerman and Schunk, 2011), teachers in the in-depth workshop realized that simply expecting students to set their own learning goals for formative assessments does not work. This requires co-regulation or, at least in the beginning, externally-facilitated regulation
(Azevedo et al., 2008). Where teachers can define the initial learning goals, students can be stimulated to play an more active role in redefining the learning goals after having identified their strengths and weaknesses (Allal, 2020) as can also be seen in the example of one of our teacher teams (Figure 3). In this same vein, one of the teachers said "I realize that simply telling students to give each other peer feedback will not do the trick". Various studies on formative assessment and SRL look for self- or peer-assessment as being present or not (Wiliam, 2011; Kippers et al., 2018; Allal, 2020). However, several studies showed that robust formative assessment implementation requires selfor peer-assessment implementation to be supported by guidelines, formats or prompts that help students in using these tools to actually regulate their learning (Panadero et al., 2012; Restrepo, 2013; Wylie and Lyon, 2015). In our study, teachers realize the importance of student-teacher interaction in the formative assessment process (Carless and Winstone, 2020). They realize that FA does not automatically bring students to regulate their own learning, but that they, as teachers, need to explicitly design a co-regulatory process that guides students in this regulatory process (McCaslin and Hickey, 2001; Azevedo et al., 2008; Panadero et al., 2019). We would argue that going through the participatory research activities in this study made teachers realize this, which is supported by their evaluations of the activities (e.g., "I now realize the importance of a thought through design using the FA cycle" and "the interplay between student and teacher behavior is my most important new insight". Exit-ticket statements of teachers in step 2, see Methods). Actively involving teachers in explicating expected students and teacher co-regulatory behavior, feeding back their own results and prompting their thinking with theoretical insights seemed helpful in elevating teachers' practical knowledge on coregulatory formative assessment in the classroom. Professional development of teachers in designing formative assessment to foster self-regulatory skills might make use of these insights. An additional added value of this approach of actively involving teachers in identifying what co-regulatory formative assessment in their classroom looks like, with their students and their subject in their context, also acknowledged that formative assessment is student- context- and discipline dependent (Winstone and Carless, 2019).

\section{Who Steers the Co-Regulatory Formative Assessment Processes?}

Andrade and Brookhart (2020) describe in extensive detail, and from a theoretical point of view, how classroom assessment can relate to all phases and areas of the regulation of learning. Though the actual interactions between students and teachers are implicit in this framework (Andrade and Brookhart, 2020). This study suggests that explicating these student-teacher interactions-using the student-teacher formative assessment cycle format (Figure 2)-is of added value for making co-regulatory formative assessment in the classroom, as well as the variations thereof, more tangible. It shows a variety of concrete co-regulatory formative assessment processes in terms of actual student-teacher interactions in the classroom. We 
identify these co-regulatory interactions to differ in the extent to which they are directed by the teacher or the student. Our data suggests that, in all instances, the teacher starts the co-regulatory formative assessment process by clarifying the initial learning goals (FA phase 1). The following FA phases show examples of student-teacher interactions that are more teacher-directed telling the student what to do (i.e., the student takes the diagnostic test, the student listens to feedback), but also many statements of students initiating the co-regulatory process (e.g., the student asks for feedback; the student chooses a task to show what he/she can do, see also Table 2), as well as statements that explicate a student-teacher or student-student dialogue (i.e., in dialogue with other students create an understanding of the success criteria; in dialogue with the teacher explore possible follow up activities). This finding suggests that also in coregulation, we can talk about a dimension from more teacherdirected to more student-directed activities (e.g., Van der Kleij et al., 2019; Brooks et al., 2021). We would argue that to foster students' development of self-regulatory skills, formative assessment classroom practices should allow for gradually making these co-regulatory interactions more student-directed, within the context of teacher defined goals. The example of phase 5 in the result section answering research question 2 exemplifies this idea of gradually making students more directing the coregulatory process.

\section{What Helps to Make Formative Assessment More Co-Regulatory?}

Next to teachers realizing the importance of purposefully designing for co-regulatory formative assessment, two other indicators are found that might facilitate formative assessment practices that incite co-regulation. These are the themes the active student and a classroom culture of making mistakes. Teacher's statements on expected student behavior refer to the student being active, or interactive, in all five phases of the formative assessment process. This active-or interactive-student aligns with the social constructivist learning theory underlying both self/co-regulation and formative assessment (Panadero et al., 2018). Thus, while this active student seems to be a given for FA that intends to stimulate SRL, actually activating students in education practice is not self-evident (Van der Kleij et al., 2019). Regarding a classroom culture of making mistakes it becomes clear in all steps of our participatory study, that teachers often refer to the importance of students and teachers being open to learning from mistakes. This becomes visible in statements on student behavior (e.g., students dare to give wrong answers, students use their mistakes to improve), statements on teacher behavior (e.g., the teacher models learning from mistakes), as well as in key intentions that several teacher teams put in the middle of the student formative assessment cycle poster (step 1), such as a growth mindset or open and honest learning attitude. Creating a collaborative culture where students and teachers are equal partners (McManus, 2009) and the focus is on a growth mindset (Clarke, 2014) is recognized as key to the implementation of formative assessment. Again, for FA and SRL theory this might be a given, though for teachers to realize this via active reflection on their own formative assessment practice in this participatory study can be regarded as key to their professional development trajectory (i.e., the FA learning network). This supports that importance of long-term participatory professional development to foster robust implementation of coregulatory formative assessment for stimulating student's self-regulated learning (see also, Brooks et al., 2021). The participatory approach as well as the formative assessment cycle as underlying theoretical framework for all activities that teachers grow to get acquainted to seem be successful in challenging teacher's formative assessment practices and underlying conceptions (DeLuca et al., 2012; DeLuca et al., 2019).

\section{Limitations}

Some aspects of this study need to be taken into account when considering the results. First, teachers participating in this study were all involved in a longitudinal formative assessment professional development trajectory (FA learning network), in which they were also familiarized with the formative assessment cycle (Gulikers and Baartman, 2017). Moreover, teachers in the in-depth workshop were only a small-and voluntary-self-selected selection of teachers, thereby certainly not representative of all teachers in the FE learning network neither of all secondary school teachers. These findings should not be treated as showing what coregulatory formative assessment in the regular secondary school classroom should look like. These findings are meant to explicate what co-regulation processes in the classroom can look like in terms of concrete student and teacher behavior. Moreover, the participatory process of illuminating the variety of co-regulatory formative assessment activities together with teachers is a key take home message of this study (See also McDowell et al., 2008).

Second, parts of this study was conducted during the COVID-lockdown period. It can be questioned if this made teachers view formative assessment and co/self-regulation differently. In a parallel study conducted with teachers of the learning network during the COVID-lockdown ${ }^{1}$ teacher's online FA practices, challenges and lessons learned were investigated. Indeed, this showed that teachers, identified new opportunities for engaging, monitoring and guiding their students in online FA. In that study we also say that teachers made more use of FA phase 5 (i.e., taking concrete follow up actions) in online FA than in their classroom FA practices. This aligns with the findings of this study that teachers were able to identify more concrete observable student behavior in this follow-up phase (see The Active Student in the CoRegulatory Formative Assessment Process Section) in online

\footnotetext{
${ }^{1}$ Veugen, M., Gulikers, J., \& Den Brok., P. (under review). Secondary school teachers' use of online formative assessment during Covid-19 lockdown: Experiences and lessons learned.
} 
FA. Also, during the lockdown period, teachers experienced new opportunities for stimulating student's self-regulatory activities as they more purposefully asked students to show evidence of their progress. On the other hand they reported that the difference between students being more or less able to self-regulate became more evident in the lockdown period. Thus, the COVID-lockdown period did inevitably change or challenge teacher's thinking about and experience with using FA and stimulating self-regulation. However, the examples and discussion during the workshops of this study did not focus on online FA and did not give the impression that teachers were only focused on, or biased because of, the lockdown situation. Actually, the online period seemed to broaden instead of hamper teacher's ideas on how to use co-regulatory $\mathrm{FA}$ to stimulate student's self-regulatory behavior. But again, it should be taken into account that all participating teachers were already experienced with using the FA cycle in their regular (face-to-face, before COVIDlockdown) classrooms.

Finally, we talk about co-regulatory student and teacher behaviors. This does not refer to the behavior area of SRL that mainly stresses time and effort planning and management, persistence and choice (Andrade and Brookhart, 2020). We use this term to stress the observable aspect of the cognition, motivation, behavior or context area of SRL that becomes visible in the classroom. Thus, the identified student or teacher behaviors in this study can refer to any of these areas. As such, this study is less sophisticated in its detailed operationalization of self- or co-regulation in the classroom. Still, we argue that it does help to make this complex and detailed framework of phases and areas of regulation more tangible in terms of

\section{REFERENCES}

Allal, L. (2020). Assessment and the Co-regulation of Learning in the Classroom. Assess. Educ. Princ. Pol. Pract. 27 (4), 332-349. doi:10.1080/ 0969594x.2019.1609411

Andersson, C., and Palm, T. (2018). Reasons for Teachers' Successful Development of a Formative Assessment Practice through Professional Development - a Motivation Perspective. Assess. Educ. Princ. Pol. Pract. 25 (6), 576-597. doi:10.1080/0969594x.2018.1430685

Andrade, H., and Brookhart, S. M. (2016). "The Role of Classroom Assessment in Supporting Self-Regulated Learning," in Assessment for Learning: Meeting the challenge of Implementation. Editors L. Allal and D. Laveault (Heidelberg: Springer), 293-309. doi:10.1007/978-3-31939211-0_17

Andrade, H. L., and Brookhart, S. M. (2020). Classroom Assessment as the Coregulation of Learning. Assess. Educ. Princ. Pol. Pract. 27 (4), 350-372. doi:10.1080/0969594x.2019.1571992

Andrade, H. (2010). "Students as the Definitive Source of Formative Assessment: Academic Selfassessment and the Self-Regulation of Learning," in Handbook of Formative Assessment. Editors H. Andrade and G. Cizek (New York: Routledge), 90-105.

Azevedo, R., Moos, D. C., Greene, J. A., Winters, F. I., and Cromley, J. G. (2008). Why Is Externally-Facilitated Regulated Learning More Effective Than SelfRegulated Learning with Hypermedia? Educ. Tech. Res. Dev. 56 (1), 45-72. doi:10.1007/s11423-007-9067-0

Black, P., and Wiliam, D. (1998). Assessment and Classroom Learning. Assess. Educ. Princ. Pol. Pract. 5 (1), 7-74. doi:10.1080/ 0969595980050102 concrete classroom student-teacher interactions in the formative assessment process.

\section{DATA AVAILABILITY STATEMENT}

The raw data supporting the conclusions of this article will be made available by the authors, without undue reservation.

\section{ETHICS STATEMENT}

Ethical review and approval was not required for the study on human participants in accordance with the local legislation and institutional requirements. The patients/participants provided their written informed consent to participate in this study.

\section{AUTHOR CONTRIBUTIONS}

All three authors have played an active role in setting up the design of this study. JG and MV have conducted and facilitated the interventions and analyzed the data. JG is the primary author of writing the paper, MV and LB have actively contributed with feedback to substantially improve the final paper.

\section{FUNDING}

This project is conducted using funding from The Dutch Ministry of Education, Culture and Science. Zaaknummer 130906.

Black, P., and Wiliam, D. (2009). Developing the Theory of Formative Assessment. Educ. Asse Eval. Acc. 21 (1), 5-31. doi:10.1007/s11092-008-9068-5

Boud, D., and Molloy, E. (2013). Rethinking Models of Feedback for Learning: the challenge of Design. Assess. Eval. Higher Educ. 38 (6), 698-712. doi:10.1080/ 02602938.2012.691462

Brandmo, C., Panadero, E., and Hopfenbeck, T. N. (2020). Bridging Classroom Assessment and Self-Regulated Learning. Assess. Educ. Princ. Pol. Pract. 27 (4), 319-331. doi:10.1080/0969594x.2020.1803589

Brooks, C., Burton, R., van der Kleij, F., Ablaza, C., Carroll, A., Hattie, J., et al. (2021). Teachers Activating Learners: The Effects of a Student-Centred Feedback Approach on Writing Achievement. Teach. Teach. Educ. 105, 103387. doi:10.1016/j.tate.2021.103387

Carless, D., and Winstone, N. (2020). Teacher Feedback Literacy and its Interplay with Student Feedback Literacy. Teach. Higher Educ., 1-14. doi:10.1080/ 13562517.2020.1782372

Clark, I. (2012). Formative Assessment: Assessment Is for Self-Regulated Learning. Educ. Psychol. Rev. 24 (2), 205-249. doi:10.1007/s10648-011-9191-6

Clarke, S. (2014). Outstanding Formative Assessment: Culture and Practice. UK: Hachette.

DeLuca, C., Chapman-Chin, A., and Klinger, D. A. (2019). Toward a Teacher Professional Learning Continuum in Assessment for Learning. Educ. Assess. 24 (4), 267-285. doi:10.1080/10627197.2019.1670056J

DeLuca, C., Luu, K., Sun, Y., and Klinger, D. A. (2012). Assessment for Learning in the Classroom: Barriers to Implementation and Possibilities for Teacher Professional Learning. AM 4, 5-29. doi:10.18296/am.0104

Gulikers, J., and Baartman, L. (2017). Doelgericht Professionaliseren: Formatieve Toetspraktijken Met Effect! Wat DOET De Docent in de klas? [Targetted Professonial Development: Formative Assessment Practices With Effect! What the Teacher DOES in the Classroom]. NRO Review Report No.: 405-15-722. 
Available at https:/www.nro.nl/sites/nro/files/migrate/Inhoudelijkeeindrapport_NRO-PPO-405-15-722_DEF.pdf (Accessed May 10, 2021).

Hattie, J., and Timperley, H. (2007). The Power of Feedback. Rev. Educ. Res. 77 (1), 81-112. doi:10.3102/003465430298487

Heritage, M., Kim, J., Vendlinski, T., and Herman, J. (2009). From Evidence to Action: A Seamless Process in Formative Assessment? Educ. Meas. Issues Pract. 28 (3), 24-31. doi:10.1111/j.1745-3992.2009.00151.x

Herman, J., Osmundson, E., Dai, Y., Ringstaff, C., and Timms, M. (2015). Investigating the Dynamics of Formative Assessment: Relationships between Teacher Knowledge, Assessment Practice and Learning. Assess. Educ. Princ. Pol. Pract. 22 (3), 344-367. doi:10.1080/0969594x.2015.1006521

Jacobs, S. (2016). The Use of Participatory Action Research within Education-Benefits to Stakeholders. World J. Educ. 6 (3), 48-55. doi:10.5430/wje.v6n3p48

Kippers, W. B., Wolterinck, C. H. D., Schildkamp, K., Poortman, C. L., and Visscher, A. J. (2018). Teachers' Views on the Use of Assessment for Learning and Data-Based Decision Making in Classroom Practice. Teach. Teach. Educ. 75, 199-213. doi:10.1016/j.tate.2018.06.015

McCaslin, M., and Hickey, D. T. (2001). "Self-regulated Learning and Academic Achievement: a Vygotskian View," in Self-regulated Learning and Academic Achievement. Editors B. J. Zimmerman and D. H. Schunk (New York: Lawrence Erlbaum Associates), 227-252.

McDowell, L., Smailes, J., Sambell, K., Sambell, A., and Wakelin, D. (2008). Evaluating Assessment Strategies through Collaborative Evidence-based Practice: Can One Tool Fit All? Innov. Educ. Teach. Int. 45 (2), 143-153. doi:10.1080/14703290801950310

McManus, S. (2009). The Attributes of Effective Formative Assessment. Washington, DC: CCSSO. Available at: http://www.ccsso.org (Accessed July 27, 2021).

Meusen-Beekman, K. D., Joosten-ten Brinke, D., and Boshuizen, H. P. A. (2016). Effects of Formative Assessments to Develop Self-Regulation Among Sixth Grade Students: Results from a Randomized Controlled Intervention. Stud. Educ. Eval. 51, 126-136. doi:10.1016/j.stueduc.2016.10.008

Miles, M. B., Huberman, A. M., and Saldaña, J. (2020). "Qualitative Data Analysis," in A Methods Sourcebook. 4th ed. (Saga: Arizona U.S.).

Nicol, D. J., and Macfarlane-Dick, D. (2006). Formative Assessment and Selfregulated Learning: a Model and Seven Principles of Good Feedback Practice. Stud. Higher Educ. 31, 199-218. doi:10.1080/03075070600572090

Panadero, E., Andrade, H., and Brookhart, S. (2018). Fusing Self-Regulated Learning and Formative Assessment: A Roadmap of where We Are, How We Got Here, and where We Are Going. Aust. Educ. Res. 45 (1), 13-31. doi:10.1007/s13384-018-0258-y

Panadero, E., Broadbent, J., Boud, D., and Lodge, J. M. (2019). Using Formative Assessment to Influence Self- and Co-regulated Learning: the Role of Evaluative Judgement. Eur. J. Psychol. Educ. 34 (3), 535-557. doi:10.1007/s10212-0180407-8

Panadero, E., Tapia, J. A., and Huertas, J. A. (2012). Rubrics and Self-Assessment Scripts Effects on Self-Regulation, Learning and Self-Efficacy in Secondary Education. Learn. Indiv. Differ. 22 (6), 806-813. doi:10.1016/j.lindif.2012.04.007

Perrenoud, P. (1998). From Formative Evaluation to a Controlled Regulation of Learning: Towards a Wider Conceptual Field. Assess. Educ. Princ. Pol. Pract. 5 (1), 85-102.
Restrepo, H. N. A. (2013). Role of Systematic Formative Assessment on Students' Views of Their Learning. PROFILE Issues Teachers' Prof. Develop. 15 (2), 165-183.

Sadler, D. R. (1989). Formative Assessment and the Design of Instructional Systems. Instr. Sci. 18, 145-165. doi:10.1007/bf00117714

Stiggins, R. J., Arter, J. A., Chappuis, J., and Chappuis, S. (2004). Classroom Assessment for Student Learning: Doing it Right-Using it Well. Portland, OR: Assessment Training Institute.

Van der Kleij, F. M., Adie, L. E., and Cumming, J. J. (2019). A Meta-Review of the Student Role in Feedback. Int. J. Educ. Res. 98, 303-323. doi:10.1016/ j.ijer.2019.09.005

Veugen, M. J., Gulikers, J. T. M., and den Brok, P. (2021). We Agree on What We See: Teacher and Student Perceptions of Formative Assessment Practice. Stud. Educ. Eval. 70, 101027. doi:10.1016/j.stueduc.2021.101027

Wiliam, D. (2014). "Formative Assessment and Contingency in the Regulation of Learning Processes," in Paper presented in a Symposium entitled Toward a Theory of Classroom Assessment as the Regulation of Learning at the annual meeting of the American Educational Research Association, Philadelphia, PA, April 3-7, 2014.

Wiliam, D. (2011). What Is Assessment for Learning? Stud. Educ. Eval. 37 (1), 3-14. doi:10.1016/j.stueduc.2011.03.001

Winstone, N., and Carless, D. (2019). Designing Effective Feedback Processes in Higher Education: A Learner-Focussed Approach. London: Routledge.

Wylie, E. C., and Lyon, C. J. (2015). The Fidelity of Formative Assessment Implementation: Issues of Breadth and Quality. Assess. Educ. Princ. Pol. Pract. 22 (1), 140-160. doi:10.1080/0969594x.2014.990416

Yin, Y., Shavelson, R. J., Ayala, C. C., Ruiz-Primo, M. A., Brandon, P. R., Furtak, E. M., et al. (2008). On the Impact of Formative Assessment on Student Motivation, Achievement, and Conceptual Change. Appl. Meas. Educ. 21 (4), 335-359. doi:10.1080/08957340802347845

Zimmerman, B. J., and Schunk, D. H. (2011). Self-regulated Learning and Academic Achievement. New York: Lawrence Erlbaum Associates, 227-252.

Conflict of Interest: The authors declare that the research was conducted in the absence of any commercial or financial relationships that could be construed as a potential conflict of interest.

Publisher's Note: All claims expressed in this article are solely those of the authors and do not necessarily represent those of their affiliated organizations, or those of the publisher, the editors and the reviewers. Any product that may be evaluated in this article, or claim that may be made by its manufacturer, is not guaranteed or endorsed by the publisher.

Copyright (๑) 2021 Gulikers, Veugen and Baartman. This is an open-access article distributed under the terms of the Creative Commons Attribution License (CC BY). The use, distribution or reproduction in other forums is permitted, provided the original author(s) and the copyright owner(s) are credited and that the original publication in this journal is cited, in accordance with accepted academic practice. No use, distribution or reproduction is permitted which does not comply with these terms. 